Provided for non-commercial research and education use. Not for reproduction, distribution or commercial use.

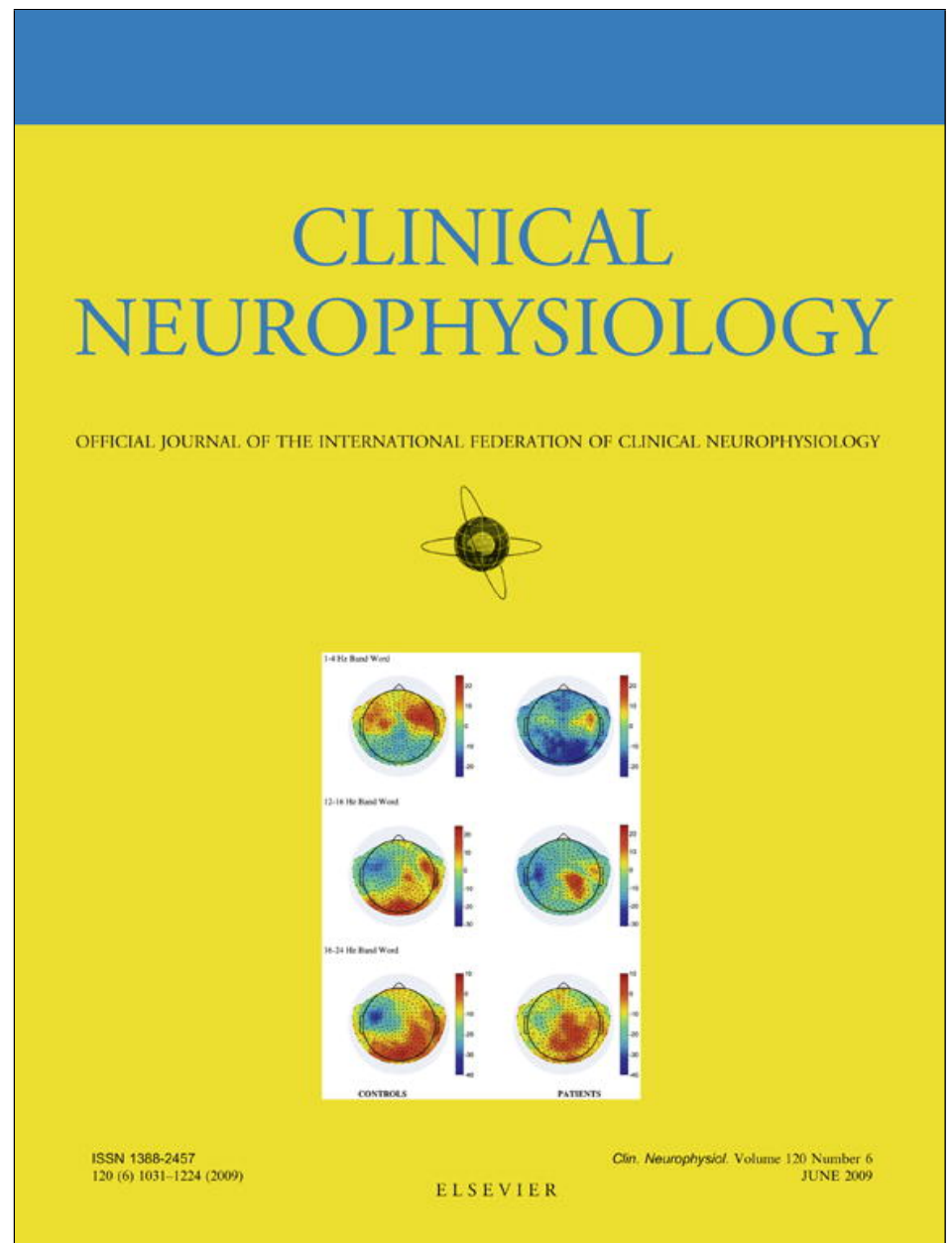

This article appeared in a journal published by Elsevier. The attached copy is furnished to the author for internal non-commercial research and education use, including for instruction at the authors institution and sharing with colleagues.

Other uses, including reproduction and distribution, or selling or licensing copies, or posting to personal, institutional or third party websites are prohibited.

In most cases authors are permitted to post their version of the article (e.g. in Word or Tex form) to their personal website or institutional repository. Authors requiring further information regarding Elsevier's archiving and manuscript policies are encouraged to visit:

http://www.elsevier.com/copyright 


\title{
Towards neurophysiological assessment of phonemic discrimination: Context effects of the mismatch negativity
}

\author{
Nina Davids ${ }^{\mathrm{a}, \mathrm{b}, *}$, Daniëlle van den Brink ${ }^{\mathrm{b}, \mathrm{c}}$, Miranda van Turennout ${ }^{\mathrm{a}, \mathrm{b}}$, Holger Mitterer ${ }^{\mathrm{d}}$, Ludo Verhoeven ${ }^{\mathrm{a}}$ \\ ${ }^{a}$ Behavioural Science Institute, Radboud University Nijmegen, The Netherlands \\ ${ }^{\mathrm{b}}$ Donders Institute for Brain, Cognition and Behaviour, Radboud University Nijmegen, The Netherlands \\ ${ }^{\mathrm{c}}$ Radboud University Nijmegen Medical Centre, The Netherlands \\ ${ }^{\mathrm{d}}$ Max Planck Institute for Psycholinguistics, The Netherlands
}

\section{A R T I C L E I N F O}

\section{Article history:}

Accepted 20 January 2009

Available online 1 May 2009

\section{Keywords:}

Mismatch negativity (MMN)

Event-related potential (ERP)

Phonemic discrimination

\begin{abstract}
A B S T R A C T
Objective: This study focusses on the optimal paradigm for simultaneous assessment of auditory and phonemic discrimination in clinical populations. We investigated (a) whether pitch and phonemic deviants presented together in one sequence are able to elicit mismatch negativities (MMNs) in healthy adults and (b) whether MMN elicited by a change in pitch is modulated by the presence of the phonemic deviants.

Methods: Standard stimuli [i] were intermixed with small, medium or large pitch deviants or with pitch deviants of the same magnitude together with small and large phonemic deviants, [y] and [u], respectively.

Results: When pitch and phonemic deviants were presented together, only the large pitch and phonemic contrasts elicited significant MMNs. When only pitch deviants were presented, the medium and large pitch contrasts elicited significant MMNs. The MMNs, in response to the medium and large pitch contrasts, were of similar magnitude across the two contexts.

Conclusions: Pitch and phonemic deviants can be tested together provided the pitch contrast is relatively large.

Significance: A combined neurophysiological test of phonemic and pitch discrimination, as measured by the MMN, is a time-effective tool that may provide valuable information about the underlying cause of poorly specified phonemic representations in clinical populations.

Crown Copyright (c) 2009 International Federation of Clinical Neurophysiology. Published by Elsevier Ireland Ltd. All rights reserved.
\end{abstract}

\section{Introduction}

A basic precursor of learning to read and spell is phonemic discrimination. If children are unable to perceive differences between phonemes, it will be difficult for them to develop phonemic representations that can be easily accessed (Manis et al., 1997). These poorly specified phonemic representations, in turn, may lead to deficits in the ability to segment phonemes, and in learning the relationship between graphemes and phonemes. Ziegler and Goswami (2005) refer to this as the availability problem. Many children with language-learning impairments (e.g., specific language impairment and dyslexia) suffer from the availability problem; that is, their language, reading and spelling problems result from poorly specified phonemic representations that are not easily accessible (Goswami, 2000; Tallal, 2004). Early detection of the

\footnotetext{
* Corresponding author. Address: Behavioural Science Institute, Radboud University Nijmegen, P.O. Box 9104, 6500 HE Nijmegen, The Netherlands. Tel.: +31 243612820 ; fax: +31243616211.

E-mail address: n.davids@pwo.ru.nl (N. Davids).
}

availability problem in clinical populations is necessary for early intervention by means of specialised training, with the purpose of minimising future reading problems. The underlying cause of the availability problem is still subject to debate (Ramus, 2001). On the one hand, theories such as the phonological deficit theory state that the problem is primarily phonological in nature. According to this theory, the processing of non-linguistic information is not affected. On the other hand, theories such as the magnocellular theory suggest that poorly specified phonemic representations are the result of a general (temporal) auditory deficit, which is caused by magnocellular deficits of the auditory system. The magnocellular theory holds that linguistic as well as non-linguistic information processing is impaired. Whether children suffer from deficits in the processing of (non-)linguistic information can be tested by means of an auditory or phonemic discrimination task. This study focusses on the optimal test to assess auditory and phonemic discrimination in clinical populations.

Assessment studies have mainly used behavioural tasks in investigating auditory and phonemic discrimination abilities in 
clinical populations (e.g., Tallal and Piercy, 1973). Most commonly, participants listen to pairs of sounds or words and are instructed to indicate by means of a verbal or motor response whether the sounds or words are the same or different. To accomplish this behavioural task, participants are required to be motivated and attentive. Moreover, participants should be able to understand the instruction as well as provide a verbal or motor response. Children from clinical populations, however, often show difficulties in meeting these requirements, and may therefore perform worse on a behavioural discrimination task than could be expected. As a consequence, proper assessment of word or sound discrimination in clinical populations should include a passive task, in which no motor or verbal response, instruction or active attention is needed. A suitable method for assessing the quality of auditory and phonemic discrimination in a passive task is the recording of event-related potentials (ERPs). An ERP is the averaged brain activity (measured in microvolt) that is elicited in response to an event, such as a visual or an auditory stimulus.

An ERP component often used for investigating auditory discrimination in a passive task is the mismatch negativity (MMN), first described by Näätänen et al. (1978). In a typical MMN task, participants passively listen to a stream of standard stimuli that are intermixed with rare occurrences of a deviant stimulus. When the ERP of the standard stimulus is subtracted from the ERP of the deviant stimulus, the MMN can be observed as a peak between 100 and $250 \mathrm{~ms}$ after stimulus onset (for a review, see Näätänen et al., 2007). The MMN has been shown to reflect the brain's automatic response to an infrequent discriminable change in a stream of frequent auditory stimuli and can be elicited by changes in frequency, intensity, spatial locus of origin, rise time, duration, phonetic structure and partial omission of an auditory stimulus (for an overview, see Näätänen, 1992). It has also been shown to be sensitive to language-specific phoneme representations (Dehaene-Lambertz, 1997; Näätänen et al., 1997). One major benefit of using the MMN in testing auditory and phonemic discrimination is that the ERP component can be elicited in the absence of attention to the auditory stimuli; besides, no instruction or a motor or verbal response is required to elicit the MMN (Näätänen, 1992). To illustrate, this ERP component has been shown to be suitable for measuring the auditory discrimination abilities of preterm infants as well as of coma patients (for an overview, see Näätänen, 1992, 2003).

Previously, the MMN has also been used to study whether children with language-learning impairments have problems with the processing of linguistic and/or non-linguistic information (e.g., Alonso-Búa et al., 2006; Lachmann et al., 2005; Meng et al., 2005; Schulte-Körne et al., 1998; Sharma et al., 2006; Uwer et al., 2002). For example, Schulte-Körne et al. (1998) found that a group of dyslexic boys, around 12 years of age, showed significantly attenuated late MMNs to synthesised speech stimuli (/da/vs. /ba/) compared to a group of control children. However, tone stimuli (1000 vs. $1050 \mathrm{~Hz}$ ) failed to show a group difference. The authors concluded that dyslexics have a specific deficit in processing speech, as opposed to non-linguistic information, such as acoustic tones. In a study by Uwer et al. (2002), two groups of children with specific language impairment (one with receptive and the other with expressive language problems; 5-10-year-olds) were compared to a control group matched on age, gender and handedness. Both these groups with specific language impairment showed significantly reduced MMNs to speech contrasts (/da/ vs. /ga/ and /ba/) compared to the control group. No significant differences in MMN amplitudes were observed in the two clinical groups. In a non-speech condition with a frequency (1000 vs. $1200 \mathrm{~Hz}$ ) and duration (175 vs. $100 \mathrm{~ms}$ ) contrast, the three groups elicited similar MMNs. Here again, it was concluded that children with specific language impairment do not have problems with simple differences between tones, but have a specific problem in discriminating speech stimuli.
The traditional paradigm used for measuring the MMN is a passive oddball paradigm, where standard stimuli are intermixed with one token of a deviant in one sequence (e.g., ...-S-S-S-S-D-S-S-S-DS-...; Näätänen, 1995; Schröger, 1998). Either a truly passive paradigm is used in which participants receive no instruction or a passive paradigm in which attention is directed away from the auditory stimuli, for instance, by showing a silent movie to the participants. Previous research has shown that ERP components can be influenced by the degree of attention that is paid to the stimuli (Clifford and Williston, 1993; Grimm et al., 2008; Rappaport et al., 1990). When the purpose is to test both auditory and phonemic discrimination in clinical populations, most studies used two different oddball series, one using tone stimuli and the other using speech stimuli (Alonso-Búa et al., 2006; Lachmann et al., 2005; Meng et al., 2005; Schulte-Körne et al., 1998; Sharma et al., 2006; Uwer et al., 2002). However, in clinical populations, it is important to obtain as much information as possible in a short test session. Therefore, for practical reasons, it would be beneficial to use a passive oddball paradigm in which standard stimuli are intermixed with multiple deviants in one sequence (e.g., ...-S-D $\mathbf{D}_{\mathbf{1}^{-}}$

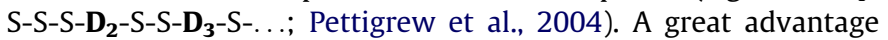
of such a paradigm, as compared to the classical paradigm with one deviant stimulus, is that it does not require a lot of extra testing time, while more information about the quality of auditory discrimination can be obtained. Recent studies have provided evidence for the usability of such a multi-deviant paradigm. They have shown that, under specific circumstances, the testing of multiple deviants in a passive oddball paradigm elicit MMNs similar to those elicited by a single deviant. For instance, Deacon et al. (1998) compared MMNs elicited by pure tones that deviated in intensity, frequency and stimulus-onset asynchrony (SOA) when each deviant was presented in a separate sequence (probability standard $=90 \%$; probability deviant $=10 \%$ ) to MMNs of all three deviants presented in one sequence (probability standard $=70 \%$; probability of each deviant $=10 \%$ ). They found that the MMNs in the multi-deviant condition were similar in magnitude to the corresponding MMNs in the single-deviant condition. These results have since been replicated by other studies (Molholm et al., 2004; Näätänen et al., 2004). In addition, Grimm et al. (2008) showed that in an active distraction paradigm with single and multiple deviants, phonemic changes elicited MMNs that were similar in magnitude.

In using the MMN in a passive paradigm to assess whether phonemic discrimination is impaired, it is of interest to test different phonemic contrasts as well as a pure acoustic contrast in one sequence. A test that includes these contrasts has several advantages. First of all, testing multiple phonemic contrasts (e.g., a small and large phonemic contrast) in one sequence provides information about whether phonemic discrimination is fully developed or not. Moreover, by testing acoustic processing as well as phonemic contrasts in one sequence, one obtains evidence whether a phonemic discrimination problem is primarily phonological in nature, as hypothesised by the phonological deficit hypothesis, or is the result of a general auditory deficit, as proposed by the magnocellular theory. However, prior to proper use of such a paradigm with multiple types of deviants in clinical populations, it is important to know whether multiple phonemic deviants and an acoustic deviant (same phoneme which differs on one acoustic dimension) in a multi-deviant design elicit MMNs in a normal population. Therefore, in the present study, we tested healthy adults on a passive oddball paradigm with three deviants: two phonemic deviants and one pitch deviant. To assess the influence of pitch magnitude in a multi-deviant design, different magnitudes of pitch deviants were used.

In this study, we investigated (a) whether phonemic and pitch changes presented in one sequence are able to elicit observable MMNs and (b) whether the MMN to pitch change is influenced 
by the presence of phonemic deviants in the same sequence. To answer the first question, a pitch contrast was presented in one sequence with two phonemic contrasts. Ideally, all contrasts should elicit observable MMNs; however, it is also conceivable that a 'more meaningful' phonemic deviant suppresses a change detection of a 'less meaningful' pitch difference. In this scenario, a pitch deviant could elicit an observable MMN when tested in a context where all stimuli, standard and deviant, are tokens of the same phoneme (i.e. with pitch variations only), whereas may fail to do so when the pitch deviant is presented together with deviants which are tokens of different phonemes (phonemic contrasts), due to grouping of the pitch deviant with the standard stimulus. To answer the second question related to context effects, the MMNs elicited by the pitch contrasts presented together with phonemic deviants in one sequence were compared to the MMNs elicited by the same pitch contrasts presented together with pitch deviants only.

\section{Experiment 1}

\subsection{Methods}

\subsubsection{Participants}

Twelve native speakers of Dutch (nine female, three male) with a mean age of 21 years (range 18-34 years) participated in the experiment. All the participants were right-handed according to an adapted version of the questionnaire by Oldfield (1971) and did not have any language, hearing or neurological disorders. The paid participants signed an informed consent in accordance with the Declaration of Helsinki.

\subsubsection{Stimuli}

The standard stimulus was a naturally produced [i] phoneme. A total of five deviants were used: three pitch deviants and two phonemic deviants. The three pitch deviants were [i] sounds which had a small, medium or large pitch difference with respect to the standard [i]. The two phonemic deviants were naturally produced $[y]$ and $[u]$, of which [y] constitutes a small phonemic difference and $[u]$ has a large phonemic difference with respect to the standard [i]. Fig. 1 shows the oscillograms of the standard as well as the large pitch deviant and the small phonemic deviant, with the pitch deviant differing from the standard only with respect to the pitch contour (middle panels) and the phonemic deviant differing with respect to the formant structure (lower panels). The standard [i] and the deviants [y] and [u] were spoken by a native Dutch female speaker and digitally recorded $(44.1 \mathrm{kHz}$, stereo) in a soundattenuated room using a Sennheiser ME62 microphone connected to a Dell D610 latitude laptop running Sony Sound Forge, a sound recording program. After recording, each stimulus was edited for precise onset and offset using a speech waveform editor (Praat, version 4.5.12). Stimuli were matched for total duration (235 ms), intensity and pitch contour (falling from $280 \mathrm{~Hz}$ at $0 \mathrm{~ms}$ to $195 \mathrm{~Hz}$ at $235 \mathrm{~ms}$ ). The pitch deviants were created by
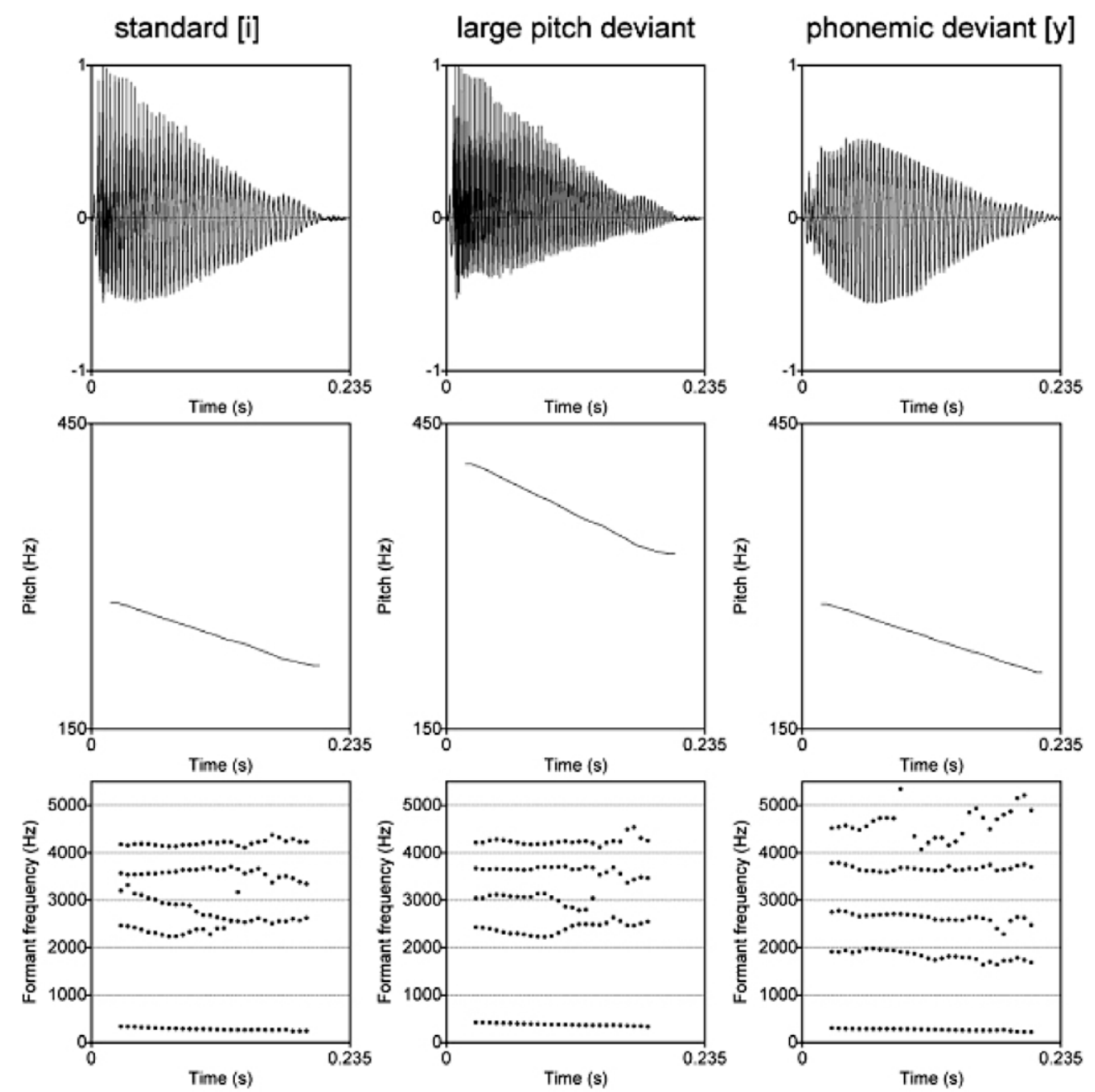

Figure 1. Stimuli used in the current study. The top row shows the oscillograms, the middle row the pitch contour and the lower row the formant tracks. The left column contains the pictures for the standard stimulus [i], the middle column for the large pitch deviant stimulus [i], and the right column for the small phonemic deviant stimulus [y]. 
Table 1

Stimuli per context condition (Experiment 1).

\begin{tabular}{llll}
\hline Context condition & Standard & Deviants & \\
\cline { 3 - 4 } & & 1 & 2 \\
\hline Small pitch + phonemic context & [i] & [i] Small pitch & [y] Small phonemic \\
Medium pitch + phonemic context & {$[\mathrm{i}]$} & [i] Medium pitch & [y] Small phonemic \\
Pitch-only context & [i] & [i] Small pitch & [i] Medium pitch Large phonemic \\
\hline
\end{tabular}

manipulating the pitch contour of the standard [i] on a semitone scale (small pitch contrast: $321-223 \mathrm{~Hz}$; medium pitch contrast: $367-256 \mathrm{~Hz}$; and large pitch contrast: $420-293 \mathrm{~Hz}$ ). The stimuli were presented at a comfortable hearing level, approximately $65 \mathrm{~dB}$.

\subsubsection{Pre-test stimuli}

To test whether the small pitch contrast was discriminable, 12 native speakers of Dutch (seven female, five male; mean age of 23 years) were asked to perform an active oddball task. The standard stimuli [i] were presented with three deviants: small, medium and large pitch deviants [i] (7\% per deviant; 40 tokens of each deviant). The participants were instructed to press a button with the right index finger when a deviant stimulus was presented. The SOA between stimuli was $800 \mathrm{~ms}$. The mean percentage correct responses to the small, medium and large pitch deviant were $60 \%(\mathrm{SD}=32 \%), 83 \%(\mathrm{SD}=18 \%)$ and $99 \%(\mathrm{SD}=2 \%)$, respectively, which corresponded to a mean $d^{\prime}$ of 2.98 ( $\mathrm{SD}=1.28$ ), 4.01 $(\mathrm{SD}=1.27)$ and $5.15(\mathrm{SD}=0.96)$, given a false alarm rate of $9 \%$ $(\mathrm{SD}=11 \%)$. This pre-test shows that all pitch deviants were perceptually distinct from the standard stimulus, but that the largest contrast was the easiest to discriminate.

\subsubsection{Design and procedure}

The participants were tested individually while seated in front of a computer screen in a soundproof, electrically shielded room. Their electroencephalogram (EEG) was recorded while they were watching a silent movie. The participants were asked to ignore the auditory stimuli. The stimuli were played with Monitor Audio speakers.

Stimuli were presented in a passive oddball paradigm with multiple deviants. Three different context conditions were created (see Table 1). Each context condition consisted of one standard stimulus and three deviant stimuli. In all context conditions, the standard stimulus was an [i] phoneme. In the first context condition, the small pitch deviant was presented together with the large and the small phonemic deviants ([y] and [u], respectively; henceforth: Small Pitch + Phonemic Context). In the second context condition, the medium pitch contrast was presented together with the two phonemic contrasts (henceforth: Medium Pitch + Phonemic Context). In the third context condition, all deviants differed from the standard only with regard to the pitch contour (henceforth: Pitch-only Context).

For every context condition, the occurrence of each deviant was $10 \%$ ( 150 tokens of each deviant and 1050 standards). The SOA between stimuli was $800 \mathrm{~ms}$, resulting in a 20-min duration of each context condition. The deviants were presented in a pseudo-random order. The three context conditions were presented to each participant. The order of the context conditions was counterbalanced between participants. Each context condition consisted of four blocks and each block started with the presentation of 15 standard stimuli. The order of the blocks was counterbalanced between participants, who had a short break between blocks.

\subsubsection{EEG recording}

The EEG was recorded from 24 electrodes using the Acticap system with $\mathrm{Ag} / \mathrm{AgCl}$ electrodes (Brain Products, Gilching, Germany).
The electrodes were placed on standard electrode sites (F7, F3, Fz, F4, F8, FC5, FC1, FCz, FC2, FC6, C3, Cz, C4, CP5, CP1, CP2, CP6, P7, P3, Pz, P4, P8, 01 and O2). All electrodes were referenced to an electrode placed at the tip of the nose. In addition, electrodes were placed at the left and the right mastoid for re-referencing during off-line analysis. To monitor vertical eye movements, electrodes were placed beneath and above the left eye. Horizontal eye movements were monitored by electrodes placed on the left and the right external canthi.

Signals were amplified with BrainAmp DC amplifiers, using a bandpass filter from 0.016 to $200 \mathrm{~Hz}$ and a sample rate of $500 \mathrm{~Hz}$. Impedances were kept below $20 \mathrm{k} \Omega$ for all electrodes. The EEG and electro-oculogram (EOG) were recorded and digitised using Brain Vision Recorder software (1.03, Brain Products, Gilching, Germany).

\subsubsection{Data analysis}

EEG data were re-referenced to the average of the left and the right mastoid and filtered with a $1-$ to $30-\mathrm{Hz}$ zero-phase-shift bandpass filter. An automatic ocular correction was performed (Gratton et al., 1983). The signal was segmented in time windows ranging from $200 \mathrm{~ms}$ before stimulus onset to $700 \mathrm{~ms}$ after stimulus onset and baseline corrected relative to a 100 -ms pre-stimulus interval. Segments with artefacts exceeding $\pm 75 \mu \mathrm{V}$ in any channel were rejected for further analysis. The remaining segments were averaged for each condition and for each participant. Difference waveforms were calculated by subtracting the ERP to the standard stimulus from the corresponding deviant stimulus. The EEG signals were analysed using Brain Vision Analyzer software (1.05.0002, Brain Products, Gilching, Germany).

Two statistical steps were performed. Firstly, we tested whether the contrasts in each context condition elicited an MMN effect. This was tested by means of a repeated-measures analysis of variance (ANOVA) applied to the mean amplitude of the difference waves at nine frontocentral electrodes in a time window of $50 \mathrm{~ms}$ centred around the grand-averaged peak of the MMN, which was determined by visual inspection of the grand-averaged MMN at Cz. The ANOVA included two within-subjects factors: Stimulus type (two levels: standard and deviant) and Electrode (nine levels: F3, Fz, F4, FC1, FCz, FC2, C3, Cz and C4). Secondly, we tested whether context effects were present for the MMNs elicited by the small and medium pitch contrasts (i.e., whether the pitch contrast was influenced by the presence of the phonemic contrasts). This was done by performing a repeated-measures ANOVA applied to the mean amplitude at the frontocentral electrodes with three within-subjects factors: Context (two levels: for the small pitch contrast: Pitch-only Context, Small Pitch + Phonemic Context; and for the medium pitch contrast: Pitch-only Context, Medium Pitch + Phonemic Context), Stimulus type (two levels: standard and deviant) and Electrode (nine levels). Greenhouse-Geisser correction for violation of sphericity assumption was applied when appropriate (Greenhouse and Geisser, 1959).

\subsection{Results}

Prior to making grand averages, we tested whether the number of artefact-free trials varied over the different stimuli. However, 
this was not the case: a repeated-measures ANOVA showed that the number of remaining trials (mean $=122$ trials across all conditions) did not differ between the stimuli $(F(3,33)=2.72, p>0.05)$. The grand-averaged difference waveforms at electrode $\mathrm{Cz}$ are presented in Fig. 2. This figure shows that the phonemic contrasts elicited large negativities, whereas the medium and large pitch contrasts elicited smaller negativities with apparent shorter peak latencies. The small pitch contrasts did not elicit any noticeable negativity.

To answer our first experimental question, we tested whether the contrasts in each context condition elicited an MMN. In Table 2 , the peak latency (measured at $\mathrm{Cz}$ ) for each contrast in each context condition is shown as well as the latency of the time windows with the corresponding mean amplitude across the nine electrodes. Moreover, the $F$-values and their significances for the main effect Stimulus type and the interaction between Stimulus type and Electrode are presented. In the Small Pitch + Phonemic Context, no peak was found for the small pitch contrast; however, both phonemic deviants elicited very clear MMNs (Fig. 2, top row). Given the absence of a peak for the small pitch deviant, no statistical test was performed for the contrast in this context condition. For the small phonemic deviant, a significant interaction between Stimulus type and Electrode emerged. The MMN in this contrast was largest at the electrodes FC1, FCz and Cz. For the large phonemic contrast, no significant interaction between Stimulus type and Electrode was found. In the Medium
Pitch + Phonemic Context, no significant MMN was obtained for the medium pitch contrast, whereas significant MMNs were found for the small and large phonemic contrasts (Fig. 2, middle row). No significant interactions between Stimulus type and Electrode emerged. In the Pitch-only Context, only the medium and large pitch contrasts elicited significant MMNs (Fig. 2, lower row). No significant interaction between Stimulus type and Electrode emerged for any of the pitch contrasts in the Pitch-only Context.

Secondly, we tested whether the small and medium pitch contrast elicited different MMNs presented in context with and without phonemic deviants. However, the small pitch contrast did not elicit any significant MMN in either the Pitch-only Context or the Small Pitch + Phonemic Context. Therefore, a context effect of the MMN was only tested for the medium pitch contrast. The negativities elicited by this contrast presented in the two different contexts are displayed in Fig. 3. A repeated-measures ANOVA revealed that there was no significant interaction between Context and Stimulus type $(F(1,11)=1.54, p>0.05)$. This indicates that the grand-averaged difference waveforms of the medium pitch contrast in the Pitch-only Context and the same contrast in the Medium Pitch + Phonemic Context did not differ statistically. For this comparison, no significant interaction between Context, Stimulus type and Electrode emerged $(F(8,88)<1, p>0.05)$.

A visual inspection of the ERPs revealed that the negativity elicited by the medium pitch contrast peaked earlier than the MMNs
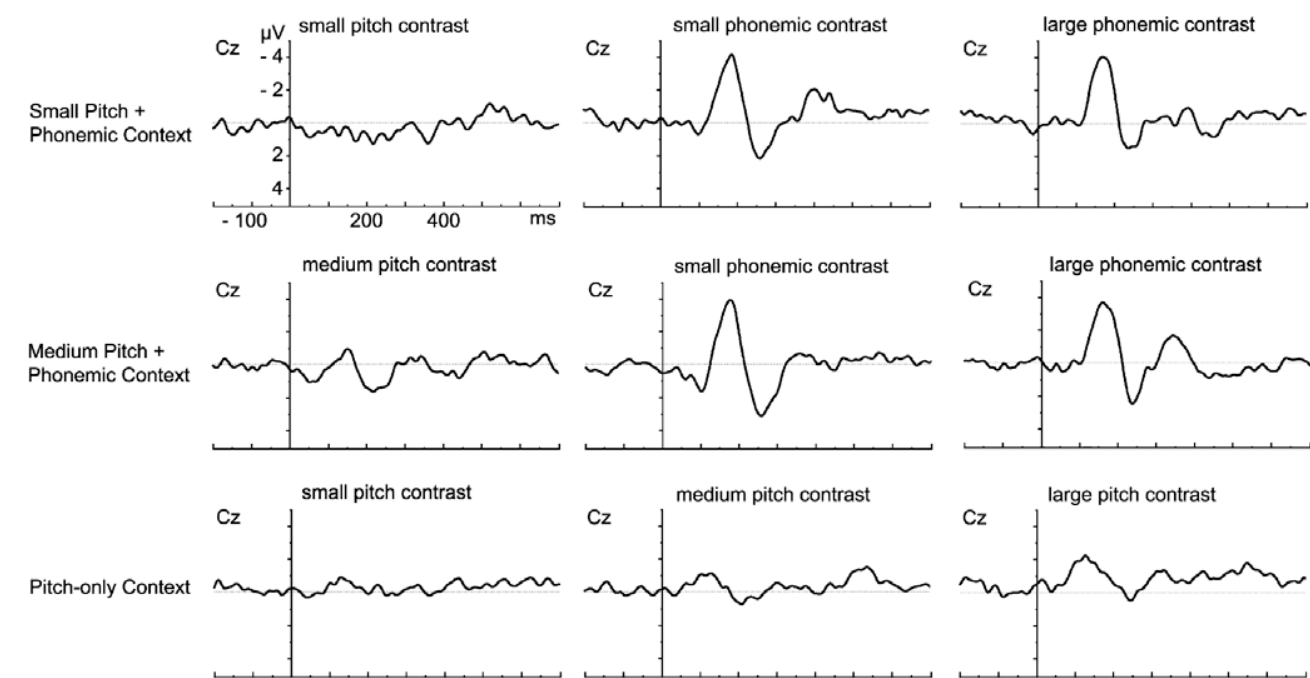

Figure 2. Grand-averaged difference waveforms (deviant-standard) at $\mathrm{Cz}$ for all contrasts per context condition in Experiment 1 . Negativity is plotted upwards.

Table 2

Relevant time windows and MMNs per context condition (Experiment 1).

\begin{tabular}{|c|c|c|c|c|c|}
\hline Contrasts per context condition & Peak latency at $\mathrm{Cz}(\mathrm{ms})$ & Time window (ms) & Amplitude $(\mu \mathrm{V})$ & Stimulus type $F(1,11)$ & Stimulus type $*$ Electrode $F(8,88)$ \\
\hline \multicolumn{6}{|l|}{ Small pitch + phonemic context } \\
\hline Small pitch & No peak & - & - & - & - \\
\hline Small phonemic & 184 & 159-209 & -2.66 & $F=24.29^{* * *}$ & $F=4.21^{*}$ \\
\hline Large phonemic & 168 & $143-193$ & -3.26 & $F=27.16^{* * *}$ & $F=3.07$ \\
\hline \multicolumn{6}{|l|}{ Medium pitch + phonemic context } \\
\hline Medium pitch & 150 & $125-175$ & -0.32 & $F<1$ & $F<1$ \\
\hline Small phonemic & 182 & $157-207$ & -2.56 & $F=18.12^{* *}$ & $F=1.46$ \\
\hline Large phonemic & 160 & $135-185$ & -3.05 & $F=36.08^{* * *}$ & $F<1$ \\
\hline \multicolumn{6}{|l|}{ Pitch-only context } \\
\hline Small pitch & 130 & $105-155$ & -0.63 & $F=2.81$ & $F=1.62$ \\
\hline Medium pitch & 128 & $103-153$ & -0.75 & $F=5.64^{*}$ & $F=1.28$ \\
\hline Large pitch & 126 & $101-151$ & -1.72 & $F=8.89^{*}$ & $F=2.45$ \\
\hline
\end{tabular}

Results of repeated measures ANOVA: ${ }^{*} p<0.05 ;{ }^{* *} p<0.01 ;{ }^{* * *} p<0.001$. 


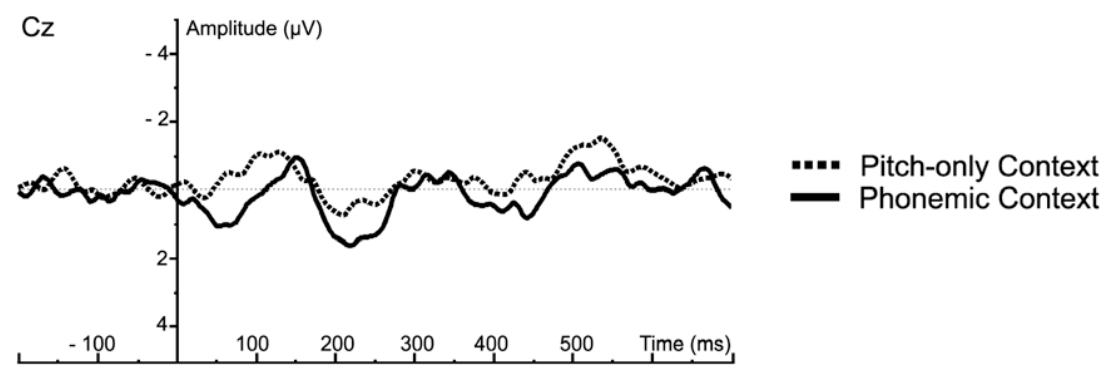

Figure 3. Grand-averaged difference waveforms for the medium pitch contrast in the Pitch-only Context (dashed line) and Medium Pitch + Phonemic Context (solid line) in Experiment 1 . Negativity is plotted upwards.

elicited by the phonemic contrasts. In the Medium Pitch + Phonemic Context, the medium pitch contrast elicited a negativity that had a peak latency of $150 \mathrm{~ms}$ at electrode $\mathrm{Cz}$, whereas the small and large phonemic MMNs at electrode $C z$ peaked at 182 and $160 \mathrm{~ms}$, respectively. A peak latency analysis, consisting of a repeated-measures ANOVA with Bonferroni correction, confirmed that the medium pitch contrast differed significantly from the small phonemic contrast $(F(1,11)=11.79, p<0.05)$, but not from the large phonemic contrast $(F(1,11)=2.78, p>0.05)$. The peak latencies of the small and large phonemic contrast did not differ significantly from each other $(F(1,11)=4.01, p>0.05)$. This may indicate that the medium pitch difference could be perceived slightly earlier than the small phonemic difference.

\subsection{Conclusions}

Our experimental question was two-fold: (a) Is it possible to elicit MMNs to phonemic and pitch deviants when they are presented in one sequence? (b) Are the MMNs to pitch deviants influenced by the presence of phonemic deviants in the same sequence? With respect to our first question, our results reveal that whereas the small and large phonemic contrasts elicited MMNs, the small and medium pitch contrasts did not. These results suggest that the phonemic deviants suppress the detection of differences in pitch, leading to the conclusion that pitch contrasts in the presence of phonemic deviants are not able to elicit MMNs. If this is indeed the case, the MMN elicited by a pitch contrast should be larger when presented in context with pitch deviants than with phonemic deviants. However, the results relating to our second experimental question showed that while the medium pitch contrast presented with pitch deviants elicited a statically significant MMN, this negativity did not differ statistically from the effect elicited by the same contrast presented in context with phonemic contrasts. These results suggest that the MMN elicited by the medium pitch contrast was not influenced by the presence of the phonemic deviants in the same sequence. Given these contradictory findings, our experimental questions cannot be answered conclusively. Therefore, we decided to conduct another experiment to test the possibility that the pitch contrasts in the presence of phonemic contrasts did not elicit MMNs simply because the pitch changes were too small (as suggested by the results in the Pitch-only Context). In Experiment 2, we investigated whether the large pitch contrast was able to elicit an MMN when it was presented in context with phonemic deviants.

\section{Experiment 2}

\subsection{Methods}

Twelve native speakers of Dutch (eight female, four male) with a mean age of 21 years (range 18-24 years) participated in the experiment. All participants were selected according to the same criteria as in Experiment 1. They signed the informed consent in accordance with the Declaration of Helsinki and were paid for participation.

\subsubsection{Stimuli}

In this experiment, the standard [i] sounds, large pitch deviant [i] and small [y] and large [u] phonemic deviants, as described in Experiment 1, were used.

\subsubsection{Design and procedure}

The design and procedure were almost identical to Experiment 1 , except that we tested only one context condition, in which standard stimuli [i] were presented with the large pitch deviant and the small and large phonemic deviants ([y] and [u], respectively). This context condition is called the 'Large Pitch + Phonemic Context'.

\subsubsection{EEG recording and analysis}

Recording settings were identical to those used in Experiment 1. To test whether all three contrasts in this context condition elicited MMNs, a repeated-measures ANOVA was applied including two within-subjects factors: Stimulus type (two levels: standard and deviant) and Electrode (nine levels: F3, Fz, F4, FC1, FCz, FC2, C3, $\mathrm{Cz}$ and (4). To test whether a context effect is present for the MMN elicited by the large pitch contrast, the MMN elicited by this contrast in the Pitch-only Context from Experiment 1 will be compared to the MMN of the large pitch contrast elicited in the Large Pitch + Phonemic Context from Experiment 2 (see Table 3). This is tested by means of a repeated-measures ANOVA of the mean amplitude at the frontocentral electrodes, including a betweensubjects factor Context (two levels: Pitch-only Context of Experiment 1 and Large Pitch + Phonemic Context of Experiment 2) and two within-subjects factors: Stimulus type (two levels: standard and deviant) and Electrode (nine levels).

\subsection{Results}

A repeated-measures ANOVA revealed that the number of remaining trials (mean $=136$ trials across all stimuli) did not differ

Table 3

Large pitch contrast tested in two context conditions.

\begin{tabular}{lllll}
\hline Context condition & Standard & Deviants & & \\
\cline { 3 - 4 } & & 1 & 2 \\
\hline Pitch-only context (Experiment 1) & [i] & [i] Small pitch & [i] Medium pitch \\
Large Pitch + phonemic context (Experiment 2) & [i] & [i] Large pitch & [y] Small phonemic & [i] Large pitch \\
\hline
\end{tabular}



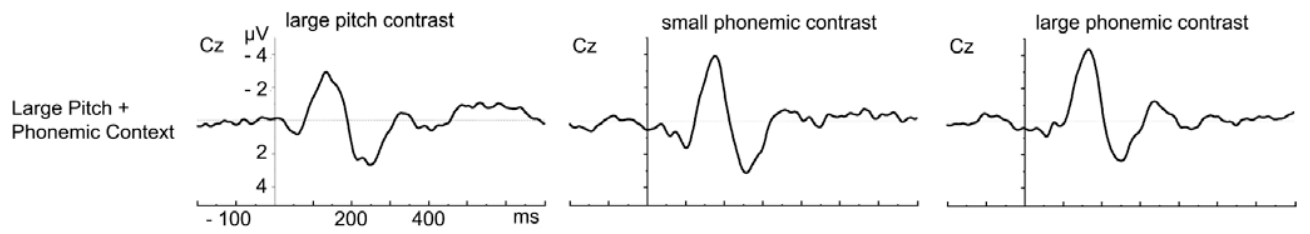

Figure 4. Grand-averaged difference waveforms (deviant-standard) at $\mathrm{Cz}$ for all contrasts in the Large Pitch + Phonemic Context in Experiment 2. Negativity is plotted upwards.

Table 4

Relevant time windows and MMNs for the Large Pitch + Phonemic Context (Experiment 2).

\begin{tabular}{|c|c|c|c|c|c|}
\hline Contrasts & Peak latency at $\mathrm{Cz}$ (ms) & Time window (ms) & Amplitude $(\mu \mathrm{V})$ & Stimulus type $F(1,11)$ & Stimulus type $*$ Electrode $F(8,88)$ \\
\hline \multicolumn{6}{|c|}{ Large pitch + phonemic context } \\
\hline Large pitch & 132 & $107-157$ & -2.23 & $F=39.16^{* * *}$ & $F=2.58$ \\
\hline Small phonemic & 174 & 149-199 & -2.96 & $F=67.09^{* * *}$ & $F=4.28^{*}$ \\
\hline Large phonemic & 166 & $141-191$ & -3.45 & $F=51.47^{* * *}$ & $F=5.85^{* *}$ \\
\hline
\end{tabular}

Results of repeated measures ANOVA: ${ }^{*} p<0.05 ;{ }^{* *} p<0.01 ;{ }^{* * *} p<0.001$.

between stimuli $(F(3,66)=1.36, p>0.05)$. The grand-averaged difference waveforms at electrode $\mathrm{Cz}$ are presented in Fig. 4. This figure shows that all three contrasts elicited large negativities in this context condition. The peak latency of the negativity elicited by the large pitch contrast appears to be shorter than the peak latencies of the effects elicited by the phonemic contrasts.

For the statistical analyses, we first tested whether all three contrasts in the Large Pitch + Phonemic Context elicited MMNs. The peak latency (measured at $\mathrm{Cz}$ ) and the latency of the $50-\mathrm{ms}$ time window for each contrast in the Large Pitch + Phonemic Context are shown in Table 4, as well as the mean amplitude across nine electrodes. In addition, the $F$-values and their significances of the main-effect Stimulus type, and the interaction between Stimulus type and Electrode are presented in this table. In the Large Pitch + Phonemic Context, all contrasts elicited significant MMNs. A significant interaction between Stimulus type and Electrode was obtained for the small and large phonemic contrasts. The MMN effect for the small phonemic contrast was largest at electrodes FC1, FCz, FC2 and Cz. The MMN effect for the large phonemic contrast was largest at electrodes FC1, FCz, FC2 and Cz.

Secondly, we tested whether there was a context effect for the MMN elicited by the large pitch contrast. The MMNs elicited by this contrast presented in the two different contexts are shown in Fig. 5. A repeated-measures ANOVA showed that there was no significant interaction between Context and Stimulus type $(F(1,22)<1, p>0.05)$. This indicated that the MMNs of the large pitch contrast in the Pitch-only Context and the one elicited in the Large Pitch + Phonemic Context did not differ statistically. For this comparison, no significant interaction emerged between Context, Stimulus type and Electrode $(F(8,176)<1, p>0.05)$.
Moreover, in the Large Pitch + Phonemic Context, the MMN of the large pitch contrast ( $132 \mathrm{~ms}$ at electrode $\mathrm{Cz}$ ) seemed to have shorter latency than the MMNs of the small and large phonemic contrasts ( 174 and $166 \mathrm{~ms}$ at electrode $\mathrm{Cz}$, respectively). A peak latency analysis, consisting of a repeated-measures ANOVA with Bonferroni correction, revealed that the MMN of the large pitch contrast peaked significantly earlier than the small and large phonemic contrasts $(F(1,11)=25.45, p<0.001$ and $F(1,11)=8.51$, $p<0.05$, respectively). In addition, the latency of the large phonemic MMN was shorter than the small phonemic MMN $(F(1,11)=11.14, p<0.05)$. This indicates that, in this experiment, the large pitch contrast could be perceived earlier than both phonemic differences.

\section{General discussion}

The present study focusses on the optimal paradigm to test auditory and phonemic discrimination by means of a passive task using the MMN in clinical populations. Such a task may serve as a means of investigating the presence and nature of the availability problem in clinical populations and whether additional training needs to be considered in the prevention of reading problems. For time-efficiency purposes, it would be beneficial to test different magnitudes of phonemic changes, as well as a pure acoustic change, simultaneously in one sequence. Therefore, we first investigated in healthy adults whether both pitch and phonemic changes were able to elicit MMNs when presented in one sequence in a passive oddball paradigm. Experiment 1 showed that pitch changes consisting of differences of approximately 2 and 5 semitones were not able to elicit MMNs when presented in a sequence

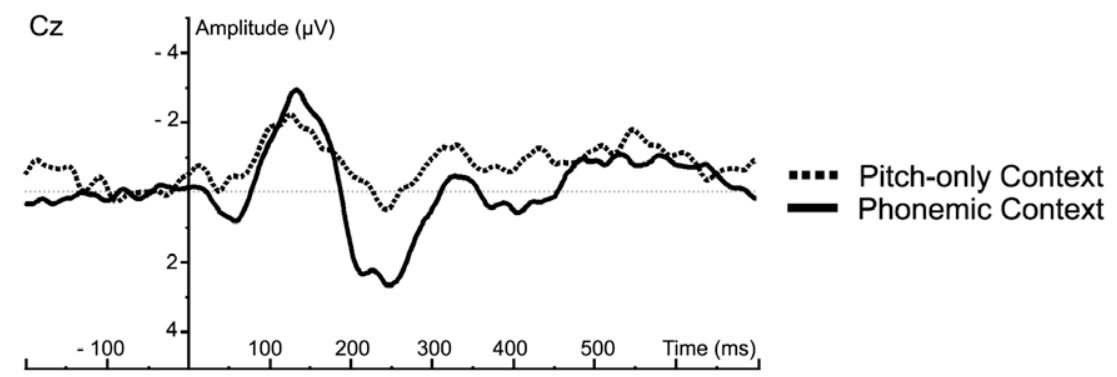

Figure 5. Grand-averaged difference waveforms for the large pitch contrast in the Pitch-only Context in Experiment 1 (dashed line) and Large Pitch + Phonemic Context in Experiment 2 (solid line). Negativity is plotted upwards. 
with two phonemic deviants. The small and large phonemic contrasts were able to elicit MMNs. However, Experiment 2 showed that the large pitch change consisting of a difference of 7 semitones was able to elicit an MMN when it was presented in context with phonemic deviants. Here again, the small and large phonemic contrasts elicited MMNs. These experiments show that when one pitch and two phonemic deviants are presented in one sequence, the phonemic contrasts are able to elicit MMNs. In contrast, pitch changes in context with phonemic deviants are only able to elicit MMNs when the difference in pitch is large enough (in our case seven semitones). If the pitch difference is too small (i.e., five semitones or less), no MMN is elicited when presented in the context of phonemic deviants. Therefore, the most suitable MMN task for testing whether the availability problem is present in children from clinical populations is the one in which a large pitch contrast is presented in combination with a small and large phonemic contrast.

The fact that the smaller pitch changes did not elicit any MMN was an unexpected finding. Our pre-test showed that the small and medium pitch contrasts were discriminable at the behavioural level. Note that the just noticeable difference (JND) for pitch is usually below 10 cents (cf. Kaernbach and Schlemmer, 2008). Given that 10 cents are 0.1 semitones, the 'small' pitch deviant of 2 semitones deviates by 20 JNDs from the standard. A possible cause of the absence of an observable MMN for the small pitch contrast may be that not all participants elicited an MMN. Individual data indeed showed that half of the participants did not show MMNs for the small pitch contrast, whereas for the large pitch contrast nine out of 12 participants elicited MMNs, which were also larger in amplitude. This means that a pitch difference, which is clearly perceivable, does not necessarily elicit an MMN. This is in contrast with what is often argued, namely, that an MMN arises to any discriminable contrast (Näätänen, 1995), but is in agreement with the findings by Pettigrew et al. (2004), as well as what is argued by Bishop (2007), revealing that easily discriminable changes often do not elicit MMNs. It is striking that there is little literature about when and why discriminable contrasts do or do not elicit MMNs. This definitely needs further investigation as it will make the application of the MMN in (clinical) populations more efficient. Furthermore, there are several studies that did find observable MMNs to pitch changes that were smaller than those used in the present study. For example, in the study of Sams et al. (1985), MMNs were elicited to simple tones with a pitch difference of about a quarter of a semitone between the standard and deviant stimulus (1000 vs. $1016 \mathrm{~Hz}$, respectively), while in our study the small difference in pitch contour consisted of more than 2 semitones (280 vs. $321 \mathrm{~Hz}$ at the beginning to $195 \mathrm{vs} .223 \mathrm{~Hz}$ at the end). The difference between the Sams et al. study and ours is that the stimuli used in the present study consisted of naturally produced phonemes containing a moving contour instead of a steady-state tone. Possibly, stimuli containing moving contours are less able to elicit MMNs, especially when small pitch contrasts are used. Future research is warranted.

Our second experimental question related to whether the MMNs to pitch changes were influenced by the presence of phonemic deviants. This was investigated by comparing the MMN to pitch change in context with phonemic deviants with the MMN to the same pitch change in context with pitch deviants only. In Experiment 1, the small pitch contrast did not elicit an MMN; therefore, the context effect was only tested for the MMN elicited by the medium pitch contrast. This MMN showed no context effect: the MMN by the medium pitch contrast in context with pitch deviants only did not differ statistically from the negativity elicited by the same contrast when it was presented together with phonemic deviants. However, the negativity itself was only statistically significant when it was presented together with pitch deviants and not when it was presented with phonemic deviants. These two findings were conflicting. Therefore, the issue was further investigated in Experiment 2. This experiment showed that the large pitch contrast elicited statistically significant MMNs both in context with phonemic deviants and in context with pitch deviants only. These MMNs, in turn, did not differ from each other. Thus, for the large pitch change, no context effect of the MMN was present. Based on these results we can conclude that the 'less meaningful' pitch contrasts are not suppressed by the presence of the 'more meaningful' phonemic deviants in the same sequence. This is in accordance with earlier studies, revealing that the presence of multiple deviants does not influence the MMNs to meaningless stimuli in a passive oddball paradigm (Deacon et al., 1998; Grimm and Schröger, 2007; Molholm et al., 2004; Näätänen et al., 2004) or to meaningful stimuli in an active distraction paradigm (Grimm et al., 2008). According to our results, these findings can be extended to the MMNs to meaningful stimuli when a passive oddball paradigm is used. However, the absence of a context effect of the MMN only stands when the acoustic difference is relatively large. In contrast to the studies mentioned above, our results seem to indicate that pitch changes can be (subtly) affected by the presence of phonemic changes: the medium pitch contrast only elicited a statistically significant MMN when it was presented with other pitch deviants and not when it was presented with phonemic deviants. This subtle influence disappeared for the large pitch contrast that elicited MMNs in both contexts. It seems that the larger the pitch contrast, the smaller the context effect.

It should be mentioned that the negativities elicited by the pitch contrasts had shorter latencies than the MMNs elicited by the phonemic contrasts. This has also been found in a study by Maurer et al. (2003) where pitch differences (1000 vs. $1030 \mathrm{~Hz}$ and $1060 \mathrm{~Hz}$ ) elicited MMNs with shorter peak latencies than phonemic contrasts (/ba/ vs. /ta/ and /da/), although this was not tested statistically. However, Corbera et al. (2006) found the reversed pattern: their frequency contrast (500 vs. $550 \mathrm{~Hz}$ ) elicited MMNs with longer latencies than their phonemic contrast (/o/ vs. /e/). Apparently, in our study, the differences in pitch could be perceived slightly earlier in time than the differences between the phonemes. Owing to the shorter latency of the pitch MMNs and the fact that we did not compare the ERPs of physically identical pitch stimuli to compute the pitch MMNs, an overlap with increased $\mathrm{N} 1$ amplitudes may have occurred. This is a result of the $\mathrm{N} 1$, which reflects acoustic characteristics of the sounds, being diminished when a stimulus is presented more often (i.e. to standard stimuli) compared to when the stimulus is presented occasionally (deviant stimuli). However, any overlap between N1 and MMN should not affect our results concerning context effects of the pitch MMNs. Consider that the critical comparison used to determine context effects of the pitch MMNs involves identical contrasts (i.e. the ERP of the same pitch deviant minus the standard stimulus) in either pitch-only or phonemic deviant context. This means (a) that a potential overlap between MMN and N1 would be identical across contexts, and (b) that the increased $\mathrm{N} 1$ effect between the contexts remains constant. This rules out the possibility that the context effects were modulated by the differential N1 effects.

Our findings indicate that auditory and phonemic discrimination abilities may be efficiently assessed at a neurophysiological level in clinical populations by measuring MMNs in a passive oddball paradigm with pitch and phonemic contrasts presented in one sequence. Using this paradigm, in a relatively short task (20 min), information can be obtained about whether clinical groups suffer from a deficit in phonemic discrimination and if this is caused by a general auditory deficit or a more specialised deficit related to phonemic processing. However, our results show that a necessary prerequisite for using such a multi-deviant paradigm is to use an 
acoustic change that is clearly perceptual in nature. Future research into the most advantageous acoustic changes will contribute a final piece to the puzzle called 'the optimal paradigm' for neuropsychological assessment of auditory and phonemic perception abilities in clinical populations.

\section{Acknowledgements}

We thank Martine Gijsel for voicing the stimuli. This study is funded by the European Union, Interreg 4-BMG-V-1=31 and Viataal (St. Michielsgestel, The Netherlands).

\section{References}

Alonso-Búa B, Díaz F, Ferraces J. The contribution of AERPs (MMN and LDN) to studying temporal vs. linguistic processing deficits in children with reading difficulties. Int J Psychophysiol 2006;59:159-67.

Bishop DV. Using mismatch negativity to study central auditory processing in developmental language and literacy impairments: where are we, and where should we be going? Psychol Bull 2007;133:651-72.

Clifford JO, Williston JS. The effects of attention and context on the spatial and magnitude components of the early responses of the event-related potential elicited by a rare stimulus. Int J Psychophysiol 1993;14:209-26.

Corbera S, Escera C, Artigas J. Impaired duration mismatch negativity in developmental dyslexia. Neuroreport 2006;17:1051-5.

Deacon D, Nousak JM, Pilotti M, Ritter W, Yang CM. Automatic change detection: does the auditory system use representations of individual stimulus features or gestalts? Psychophysiology 1998;35:413-9.

Dehaene-Lambertz G. Electrophysiological correlates of categorical phoneme perception in adults. Neuroreport 1997;8:919-24.

Goswami U. Phonological representations, reading development and dyslexia: towards a cross-linguistic theoretical framework. Dyslexia 2000;6:133-51.

Gratton G, Coles MG, Donchin E. A new method for off-line removal of ocular artifact. Electroencephalogr Clin Neurophysiol 1983;55:468-84.

Greenhouse SW, Geisser S. On methods in the analysis of profile data. Psychometrika 1959;24:95-112.

Grimm S, Schröger E. The processing of frequency deviations within sounds: evidence for the predictive nature of the Mismatch Negativity (MMN) system. Restor Neurol Neurosci 2007:25:241-9.

Grimm S, Schröger E, Bendixen A, Bass P, Roye A, Deouell LY. Optimizing the auditory distraction paradigm: behavioral and event-related potential effects in a lateralized multi-deviant approach. Clin Neurophysiol 2008;119:934-47.

Kaernbach C, Schlemmer K. The decay of pitch memory during rehearsal. J Acoust Soc Am 2008;123:1846-9.

Lachmann T, Berti S, Kujala T, Schröger E. Diagnostic subgroups of developmental dyslexia have different deficits in neural processing of tones and phonemes. Int J Psychophysiol 2005;56:105-20.

Manis FR, McBride-Chang C, Seidenberg MS, Keating P, Doi LM, et al. Are speech perception deficits associated with developmental dyslexia? J Exp Child Psychol 1997:66:211-35.
Maurer U, Bucher K, Brem S, Brandeis D. Development of the automatic mismatch response: from frontal positivity in kindergarten children to the mismatch negativity. Clin Neurophysiol 2003;114:808-17.

Meng X, Sai X, Wang C, Wang J, Sha S, Zhou X. Auditory and speech processing and reading development in Chinese school children: behavioural and ERP evidence. Dyslexia 2005;11:292-310.

Molholm S, Gomes H, Lobosco J, Deacon D, Ritter W. Feature versus gestalt representation of stimuli in the mismatch negativity system of 7- to 9-year-old children. Psychophysiology 2004;41:385-93.

Näätänen R. Attention and brain function. Hillsdale, NJ: Lawrence Erlbaum Associates; 1992.

Näätänen R. The mismatch negativity: a powerful tool for cognitive neuroscience. Ear Hear 1995;16:6-18.

Näätänen R. Mismatch negativity: clinical research and possible applications. Int Psychophysiol 2003;48:179-88.

Näätänen R, Gaillard AW, Mantysalo S. Early selective-attention effect on evoked potential reinterpreted. Acta Psychol 1978;42:313-29.

Näätänen R, Lehtokoski A, Lennes $M$, Cheour M, Huotilainen $M$, Iivonen $A$, et al. Language-specific phoneme representations revealed by electric and magnetic brain responses. Nature 1997;385:432-4

Näätänen R, Paavilainen P, Rinne T, Alho K. The mismatch negativity (MMN) in basic research of central auditory processing: a review. Clin Neurophysio 2007;118:2544-90.

Näätänen R, Pakarinen S, Rinne T, Takegata R. The mismatch negativity (MMN): towards the optimal paradigm. Clin Neurophysiol 2004:115:140-4.

Oldfield RC. The assessment and analysis of handedness: the Edinburgh inventory. Neuropsychologia 1971;9:97-113.

Pettigrew CM, Murdoch BE, Ponton CW, Finnigan S, Alku P, Kei J, et al. Automatic auditory processing of English words as indexed by the mismatch negativity, using a multiple deviant paradigm. Ear Hear 2004;25:284-301.

Ramus F. Dyslexia. Talk of two theories. Nature 2001;412:393-5.

Rappaport M, Clifford JO, Winterfield KM. P300 response under active and passive attentional states and uni- and bimodality stimulus presentation conditions. J Neuropsychiatry Clin Neurosci 1990;2:399-407.

Sams M, Paavilainen P, Alho K, Näätänen R. Auditory frequency discrimination and event-related potentials. Electroencephalogr Clin Neurophysiol 1985;62:437-48

Schröger E. Measurement and interpretation of the mismatch negativity. Behav Res Methods Instrum Comput 1998:30:131-45.

Schulte-Körne G, Deimel W, Bartling J, Remschmidt H. Auditory processing and dyslexia: evidence for a specific speech processing deficit. Neuroreport 1998;9:337-40.

Sharma M, Purdy SC, Newall P, Wheldall K, Beaman R, Dillon H. Electrophysiology and behavioural evidence of auditory processing deficits in children with reading disorder. Clin Neurophysiol 2006;117:1130-44.

Tallal P. Improving language and literacy is a matter of time. Nat Rev Neurosci 2004;5:721-8.

Tallal P, Piercy M. Developmental aphasia: Impaired rate of non-verbal processing as a function of sensory modality. Neuropsychologica 1973;11:389-98.

Uwer R, Albrecht R, von-Suchodoletz W. Automatic processing of tones and speech stimuli in children with specific language impairment. Dev Med Child Neurol 2002;44:527-32.

Ziegler JC, Goswami U. Reading acquisition, developmental dyslexia, and skilled reading across languages: a psycholinguistic grain size theory. Psychol Bull $2005 ; 131: 3-29$. 